\title{
An open code pledge for the neuroscience community
}

\author{
Cooper A. Smout ${ }^{1,2, *}$, Dawn Liu Holford ${ }^{3}$, Kelly G. Garner ${ }^{2,4,5}$, Manuel Illanes $^{6}$, $\underline{\text { Paula Andrea }}$ \\ $\underline{\text { Martinez }}^{7,8}, \underline{\text { Megan E.J. Campbel1 }}^{9,10}$, Ibrahim Khormi ${ }^{9}{ }^{10}$, Dylan G. E. Gomes ${ }^{11}$, Johanna M. M.

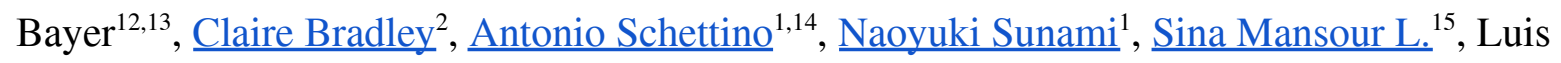 \\ Pedro Coelho ${ }^{16,17, *}$
}

1. Institute for Globally Distributed Open Research and Education (IGDORE)

2. Queensland Brain Institute, The University of Queensland, Brisbane, Australia

3. School of Psychological Science, University of Bristol, UK

4. School of Psychology, The University of Queensland, Brisbane, Australia

5. School of Psychology, University of Birmingham, UK

6. Experimental Research Unit, Bolivian Catholic University San Pablo

7. Australian Research Data Commons, Australia

8. Research Software Alliance

9. University of Newcastle, Newcastle, Australia

10. Hunter Medical Research Institute, Newcastle, Australia

11. Cooperative Institute for Marine Resources Studies, Hatfield Marine Science Center, Oregon State University, Newport, OR, United States

12. Orygen, Parkville, Australia

13. Centre for Youth Mental Health, The University of Melbourne, Australia

14. Erasmus Research Services, Erasmus University Rotterdam, Rotterdam, the Netherlands

15. Department of Biomedical Engineering, The University of Melbourne, Australia

16. Institute of Science and Technology for Brain-Inspired Intelligence, Fudan University, Shanghai, China

17. MOE Key Laboratory of Computational Neuroscience and Brain-Inspired Intelligence, and MOE Frontiers Center for Brain Science, China

* corresponding authors: cooper.smout@gmail.com, luis@luispedro.org

The authors declare that there are no conflicts of interest. Antonio Schettino is employed at Erasmus University Rotterdam as Coordinator Open Science. The employer had no role in project design, literature review, writing of the paper, and/or decision to submit for publication. This project has received funding from the European Union's Horizon 2020 research and 
innovation programme under the Marie Sklodowska-Curie grant agreement No 796329, awarded to Kelly Garner. 


\begin{abstract}
Sharing of research code would greatly benefit neuroscience, but this practice is hampered by a collective action problem. Since the development of the internet, conditional pledge platforms (e.g., Kickstarter) have increasingly been used to solve globally-dispersed collective action problems (Hallam, 2016). However, this strategy has yet to be implemented within academia. In this brief paper, we introduce a general purpose conditional pledge platform for the research community: Project Free Our Knowledge. We highlight a new conditional pledge campaign that was initiated at Brainhack 2021 and aims to motivate a critical mass of neuroscientists to share their research code. Crucially, this commitment activates only when a user-defined threshold of support is reached. We conclude by sharing our vision for how the research community could use collective action campaigns to create a sustained, evidence-based movement for social change in academia.
\end{abstract}




\section{The collective action problem in academia}

Neuroscience relies on research code for experimentation, data cleaning and analysis. If individual researchers share their code openly, the collective neuroscience community benefits through verifiability, greater reproducibility and less unnecessary duplication (Eglen et al., 2017; Riquelme \& Gjorgjieva, 2021). Most neuroscientists do not share their code, however, because non-article research outputs are not currently rewarded in academia and thus individuals may perceive sharing code as a risk to their career (Eglen et al., 2017; LeVeque, 2013). The global neuroscience community is thus trapped in a collective action problem (Olson, 1971): the community is failing to provide a public good (open source code) due to competing interests at the individual level (Coelho, 2013).

\section{A new solution: Conditional pledges}

Project Free Our Knowledge (FOK) aims to solve this class of problems by organising collective action between researchers. FOK facilitates conditional pledges: commitments to adopt open and reproducible research practices on the condition that $N$ researchers pre-agree to take action (Figure 1). If the threshold is met, the pledging community takes action together, thus empowering individuals to achieve their common aims. If the threshold is not met, however, no further action is required, thus mitigating any risks associated with solitary action. FOK draws from prior conditional pledge platforms that have helped millions of globally-dispersed individuals overcome collective action problems in the economic (e.g., Kickstarter), cultural (e.g., Collaction) and political spheres (e.g. Pledgebank; Hallam, 2016), and seeks to bring a comparable but tailored solution to the research community.

\section{The open source and citable code pledge}

During Brainhack 2021, we developed a conditional pledge campaign that aims to motivate neuroscientists to share their code in a public repository (e.g., Zenodo, OSF) with a persistent identifier (e.g., digital object identifier; DOI). Coinciding with the present publication, we hereby invite all neuroscientists to take the Open source and citable code pledge: 
"I pledge to share the code underlying all of my future publications in a citable repository."

Pledgers can either begin sharing their code immediately (c.f., Gleeson et al., 2017) or wait until $N$ neuroscientists have taken the pledge (conditional pledge; see the FOK website for details).

\section{Next steps}

We intend to promote the campaign through various communication channels and strategies (e.g., ambassador network; Figure 1A). If the campaign reaches the threshold, pledgers will be publicly listed and directed to take action together (Figure 1B). We will then analyse the campaign outcomes (e.g., pledge compliance, citation metrics) and use this information to maximise the impact of future campaigns that target a range of open and reproducible research practices in different research fields (Figure 1C-D). In short, we seek to establish a sustained, evidence-based movement for social change in academia (Figure 1E). We hereby invite all researchers to join us in this vision by proposing new campaigns and taking pledges on the FOK website today. 

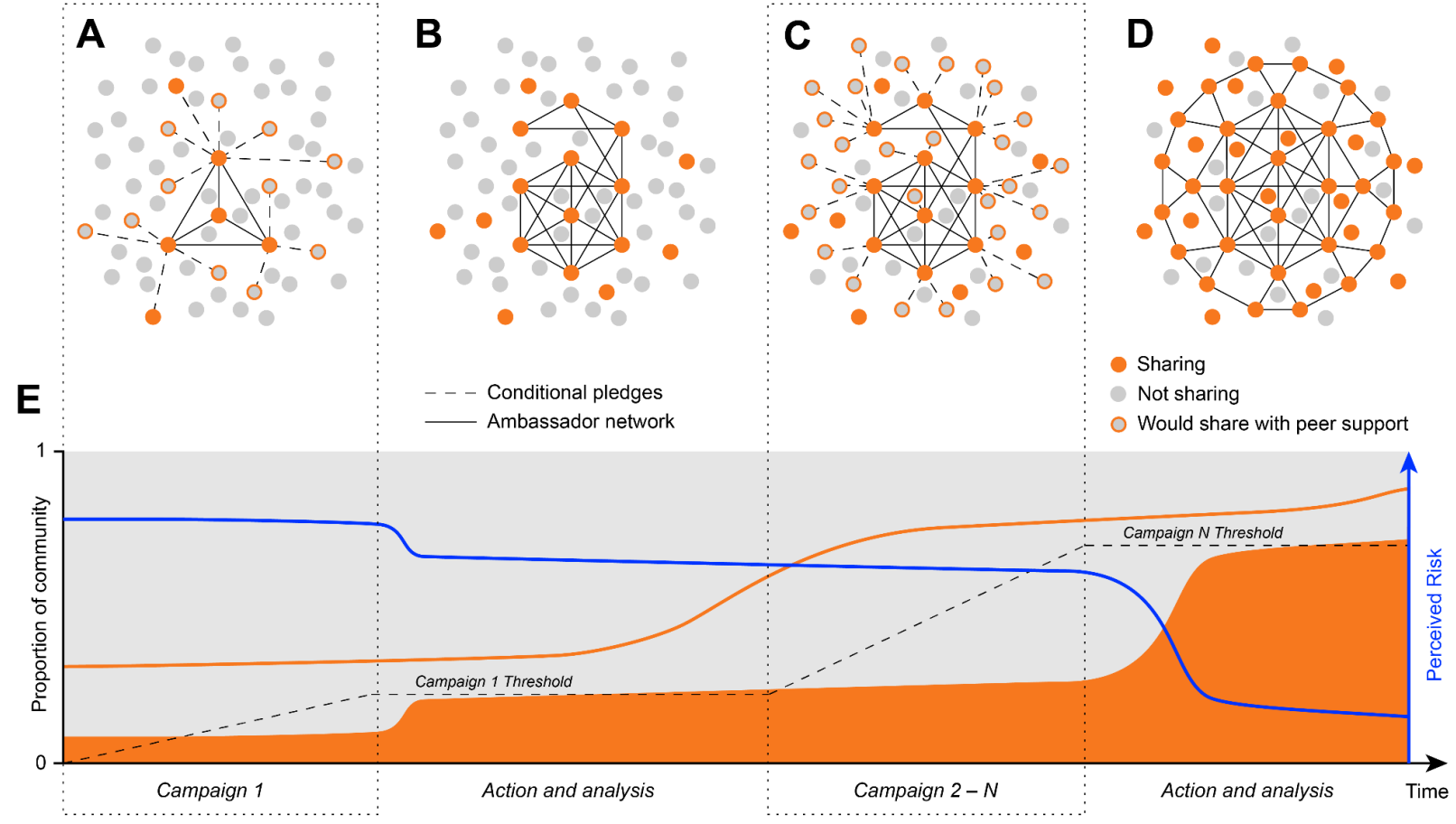

Figure 1. Using conditional pledges to solve collective action problems in academia (e.g., code-sharing in neuroscience). (A) Current state. A minority of neuroscientists share code (orange dots). A larger latent group (Olson, 1971) would be willing to share code if they were not alone in doing so (orange-bordered dots). Ambassador network (solid black lines) promotes the campaign. Researchers sign conditional pledges (dashed lines) to act when a predetermined threshold is met. (B) Campaign reaches the threshold. Pledgers share their code (orange dots). New ambassadors join the network (solid lines). Campaign outcomes are analysed and used to inform the design of a follow-up campaign (or multiple campaigns). (C) Follow-up campaign is launched. Ambassadors promote the campaign and a larger cohort of researchers sign conditional pledges. (D) Future state. The majority of researchers share code (orange dots), flipping the social norm so that individuals who do not share face social pressure (grey dots). (E) Developing a sustained movement for social change. At any one point, the number of researchers who would be willing to share code if they had peer support (orange line) is greater than the number of researchers currently sharing (orange patch). Each campaign serves to connect this latent group via conditional pledges (dashed lines) and coordinate collective action once the target threshold is reached. Successive campaigns leverage the established community to reach greater thresholds and/or motivate new behaviours (e.g., 'FAIR' code; Chue Hong et al., 2021; Goble et al., 2020; Lamprecht et al., 2020). Perceived risk to individuals' careers is inversely related to the number of people sharing (blue line). Following the $N$ th campaign, sharing code becomes normative and perceived risks are dramatically reduced (note that campaigns can also evolve in parallel, rather than sequentially as pictured here). 


\section{References}

Chue Hong, N. P., Katz, D. S., Barker, M., Lamprecht, A.-L., Martinez, C., Psomopoulos, F. E., Harrow, J., Castro, L. J., Gruenpeter, M., Martinez, P. A., Honeyman, T., Struck, A., Lee, A., Loewe, A., van Werkhoven, B., Jones, C., Garijo, D., Plomp, E., Genova, F., ... Yehudi, Y. (2021). FAIR Principles for Research Software (FAIR4RS Principles). Research Data Alliance, 32. https://doi.org/10.15497/RDA00065

Coelho, L. P. (2013, May 6). People are Right Not to Share Scientific Code... but we are wrong to let them get away with it. Meta Rabbit. https://metarabbit.wordpress.com/2013/05/06/people-are-right-not-to-share-scientific-code/

Eglen, S. J., Marwick, B., Halchenko, Y. O., Hanke, M., Sufi, S., Gleeson, P., Silver, R. A., Davison, A. P., Lanyon, L., Abrams, M., Wachtler, T., Willshaw, D. J., Pouzat, C., \& Poline, J.-B. (2017). Toward standard practices for sharing computer code and programs in neuroscience. Nature Neuroscience, 20(6), 770-773. https://doi.org/10.1038/nn.4550

Gleeson, P., Davison, A. P., Silver, R. A., \& Ascoli, G. A. (2017). A Commitment to Open Source in Neuroscience. Neuron, 96(5), 964-965. https://doi.org/10.1016/j.neuron.2017.10.013

Goble, C., Cohen-Boulakia, S., Soiland-Reyes, S., Garijo, D., Gil, Y., Crusoe, M. R., Peters, K., \& Schober, D. (2020). FAIR Computational Workflows. Data Intelligence, 2(1-2), 108-121. https://doi.org/10.1162/dint_a_00033

Hallam, R. (2016). How the internet can overcome the collective action problem: Conditional commitment designs on Pledgebank, Kickstarter, and The Point/Groupon websites. Information, Communication \& Society, 19(3), 362-379. https://doi.org/10.1080/1369118X.2015.1109696

Lamprecht, A.-L., Garcia, L., Kuzak, M., Martinez, C., Arcila, R., Martin Del Pico, E., Dominguez Del Angel, V., van de Sandt, S., Ison, J., Martinez, P. A., McQuilton, P., Valencia, A., Harrow, J., Psomopoulos, F., Gelpi, J. L., Chue Hong, N., Goble, C., \& Capella-Gutierrez, S. (2020). Towards FAIR principles for research software. Data Science, 3(1), 37-59. https://doi.org/10.3233/DS-190026

LeVeque, R. J. (2013, April 1). Top Ten Reasons To Not Share Your Code (and why you should anyway). Siam News. https://sinews.siam.org/Details-Page/top-ten-reasons-to-not-share-your-code-and-why-you-should-anyway

Olson, M. (1971). The Logic of Collective Action: Public Goods and the Theory of Groups, Second Printing with a New Preface and Appendix. Harvard University Press. https://doi.org/10.2307/j.ctvjsf3ts

Riquelme, J. L., \& Gjorgjieva, J. (2021). Towards readable code in neuroscience. Nature Reviews Neuroscience, 22(5), 257-258. https://doi.org/10.1038/s41583-021-00450-y 